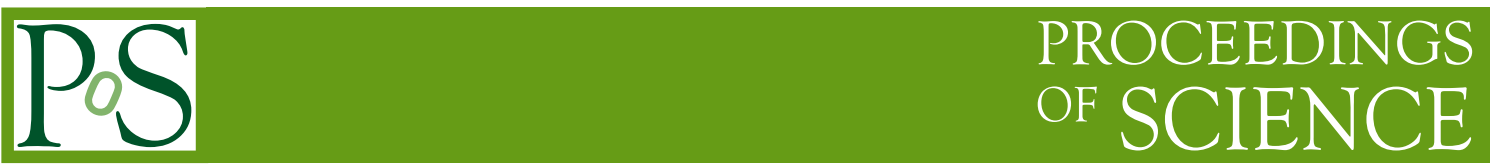

\title{
Nuclear models for light systems
}

\author{
Pierre Descouvemont ${ }^{* \dagger}$ \\ Physique Nucléaire Théorique et Physique Mathématique, CP229, Université Libre de \\ Bruxelles, B1050 Brussels, Belgium \\ E-mail: pdesc@ulb.ac.be
}

\section{Marianne Dufour}

IReS Bat27, IN2P3-CNRS/Université Louis Pasteur BP28, F67037 Strasbourg Cedex 2 - France

E-mail: Marianne.Dufour@IReS.in2p3.fr

\section{Natalia K. Timofeyuk}

Department of Physics, University of Surrey, Guildford, Surrey GU2 7XH, UK

E-mail: N.Timofeyuk@surrey.ac.uk

\begin{abstract}
Different theoretical models adapted to low-energy reactions are briefly described. We emphasize on the microscopic approach, where all information is obtained from a nucleon-nucleon interaction. A first application deals with the ${ }^{14} \mathrm{C}(\mathrm{n}, \gamma){ }^{15} \mathrm{C}$ reaction, where we use the Asymptotic Normalization Constant (ANC) method. By using recent data on ${ }^{14} \mathrm{O}+\mathrm{p}$ elastic scattering combined with a microscopic analysis, we show evidence that existing direct and some indirect data on ${ }^{14} \mathrm{C}(\mathrm{n}, \gamma){ }^{15} \mathrm{C}$ are inconsistent with charge symmetry. We also present new results on the ${ }^{18} \mathrm{~F}(\mathrm{p}, \alpha){ }^{15} \mathrm{O}$ reaction, obtained in a microscopic cluster model. We point out that $1 / 2^{+}$resonances, generally disregarded, may play a role. This property reduces the uncertainties associated with the $3 / 2^{+}$contribution. The relevant $1 / 2^{+}$states have been observed in ${ }^{19} \mathrm{~F}$, but have not been searched for in ${ }^{19} \mathrm{Ne}$. An elastic-scattering experiment near $E_{c m} \approx 1.5 \mathrm{MeV}$ is suggested.
\end{abstract}

International Symposium on Nuclear Astrophysics - Nuclei in the Cosmos - IX

25-30 June 2006

CERN

\footnotetext{
*Speaker.

${ }^{\dagger}$ Directeur de Recherches FNRS.
} 


\section{Introduction}

Nuclear astrophysics plays a crucial role in the understanding of nucleosynthesis in the universe [1]. Nuclear reactions determine the stellar evolution and are the major energy source in stars. Current modelisations of star evolution require a very large amount of data, which remains a challenge for nuclear physicists. Experimental investigations meet two main limitations. $(i)$ For charged-particle reactions, relevant energies are much lower than the Coulomb barrier, which makes the cross sections too small to be measured. Recent technological developments allow measurements at stellar energies for some reactions with low charges, such as ${ }^{3} \mathrm{He}\left({ }^{3} \mathrm{He}, 2 \mathrm{p}\right) \alpha$ [2], but for most important reactions the experimental lower limit is far above the Gamow energy. (ii) Investigations of explosive burning are fairly sensitive to reactions involving unstable nuclei. Development of radioactive ion beams [3] in many laboratories provides useful information on such reactions, but much work remains to be carried out.

In view of these limitations, theoretical models $[4,5]$ of nuclear reactions appear to be a necessary complement to experimental studies. This is obvious for light systems, where there is no systematics; each reaction presents its own peculiarities. Theoretical calculations can be done at any energy, and are not restricted by the instability of the nuclei. On the other hand, indirect methods [6] are known to provide useful and complementary information. Various methods, such as the Coulomb dissociation [7], the Trojan Horse [8], or the Asymptotic Normalization Method (ANC) [9] have been developed and successfully applied to different reactions. It is of course impossible for any model to cover the thousands of reactions involved in modern codes of stellar evolution. However, a limited number of reactions, especially in the low-mass range, play a major role, and deserve special attention.

We focus here on light systems, where the level density is low (at most a few levels per $\mathrm{MeV}$ ). This is of course crucial in theories where each level must be considered individually. We present a short overview of different models, and two recent applications. The former deals with the ${ }^{14} \mathrm{C}(\mathrm{n}, \gamma){ }^{15} \mathrm{C}$ reaction where the ANC method has been used to discriminate between different conflicting data sets [10]. The latter concerns the ${ }^{18} \mathrm{~F}(\mathrm{p}, \alpha){ }^{15} \mathrm{O}$ reaction, which attracted attention of many groups in recent years [11].

\section{Theoretical models}

\subsection{General discussion}

Theoretical models can be roughly classified in three categories:

(i) Models involving adjustable parameters, such as the $R$-matrix [12] method; parameters are fitted to the available experimental data and the cross sections are extrapolated down to astrophysical energies. These fitting procedures of course require the knowledge of data, which are sometimes too scarce for a reliable extrapolation.

(ii) Models where the cross section is determined from the wave functions of the system. The potential model [13], the Distorted Wave Born Approximation (DWBA) [14], and microscopic cluster models [15] are, in principle, independent of experimental data. More realistically, these models depend on some physical parameters, such as a nucleus-nucleus or a nucleon-nucleon interaction which can be reasonably determined from experiment only. The microscopic Generator Coordinate 
Method (GCM) provides a "basic" description of a $A$-nucleon system, since the whole information is obtained from a nucleon-nucleon interaction. Since this interaction is nearly the same for all light nuclei, the predictive power of the GCM is important. For few-body systems, "ab initio" calculations are available [16], but for $A>4$ the treatment of the scattering wave functions is still done within the potential model.

(iii) Models mentioned in $(i)$ and (ii) can be used for low level-density nuclei only. This condition is fulfilled in most of the reactions involving light nuclei $(A \leq 20)$. However when the level density near threshold is large (i.e. more than a few levels per $\mathrm{MeV}$ ), statistical models are better adapted. Statistical models, such as the Hauser-Feshbach theory [17] can be used in a systematic way.

\subsection{The $R$-matrix method}

Owing to the very low cross sections, one of the main problems in nuclear astrophysics is to extrapolate the available data down to stellar energies[1,4]. Several models, such as the potential model or microscopic approaches, are widely used for that purpose. However, they are in general not flexible enough to account for the data with a high accuracy. A simple way to extrapolate the data is to use a polynomial approximation. This is usually used to investigate electron screening effects, where the cross section between bare nuclei is derived from a polynomial extrapolation of high-energy data. This polynomial approximation, although very simple, is not based on a rigorous treatment of the energy dependence of the cross section. In the $R$-matrix method, the energy dependence of the cross sections is obtained from Coulomb functions, as expected from the Schrödinger equation. The goal of the $R$-matrix method [12] is to parameterize some experimentally known quantities, such as cross sections or phase shifts, with a small number of parameters, which are then used to extrapolate the cross section down to astrophysical energies.

The $R$-matrix framework assumes that the space is divided into two regions: the internal region (with radius $a$ ), where the nuclear force is dominant, and the external region, where the interaction between the nuclei is governed by the Coulomb force only. Although the $R$-matrix parameters do depend on the channel radius $a$, the sensitivity of the cross section with respect to its choice is quite weak. Many applications have been treated by the $R$-matrix method, in particular the ${ }^{12} \mathrm{C}(\alpha, \gamma){ }^{16} \mathrm{O}$ reaction [18].

\subsection{The potential model}

The potential model is fairly simple to use, and has been applied to many reactions in lowenergy nuclear physics (see Ref. [13] and references therein). The basic assumptions of the potential model are: $(i)$ the nucleon-nucleon interaction leads to a nucleus-nucleus force, which depends on the relative coordinate only; (ii) the wave functions of the unified nucleus can be described by a cluster structure with $A_{1}+A_{2}$ nucleons; (iii) the internal structure of the nuclei does not play any role.

The Schrödinger equation is solved numerically for most potentials. Usually this equation is integrated by using the Numerov method [19]. For bound states, the energy is obtained by requiring the wave function to be squared integrable. For scattering states, the numerical solution is fitted, at large distances, to a linear combination of Coulomb functions, which provides the phase shift. 


\subsection{Microscopic cluster models}

The information is derived from a $A$-body Hamiltonian

$$
H=\sum_{i=1}^{A} T_{i}+\sum_{i<j}^{A} V_{i j}
$$

where $T_{i}$ is the kinetic energy of nucleon $i$, and $V_{i j}$ a nucleon-nucleon interaction. In the cluster approximation [15], the wave functions are defined from the cluster wave functions $\phi_{1}$ and $\phi_{2}$ of the colliding nuclei. The total wave function reads, in a schematic notation

$$
\Psi=\mathscr{A} \phi_{1} \phi_{2} g(\rho)
$$

where $g(\rho)$ is the relative function depending on the relative coordinate $\rho$; it is determined from the Schrödinger equation. In (2.2), $\mathscr{A}$ is the antisymmetrization operator which ensures the Pauli principle to be satisfied. Projection over good quantum numbers is performed exactly. The reliability on the model mostly depends on the accuracy of the internal wave functions $\phi_{1}$ and $\phi_{2}$. Many efforts have been done to go beyond the simple shell model approximation: multicluster description [20], monopole distortion [21] and extended shell model developments [22] aim at improving the definition (2.2). In practice the radial wave function is expanded on a set of displaced gaussian functions, centered at different locations. This is the starting point of the Generator Coordinate Method (GCM - see Ref. [15] for detail). Many applications have been considered so far, not only in nuclear astrophysics, but also in nuclear physics, where a microscopic approach is well adapted to the spectroscopy of exotic nuclei.

\subsection{Indirect methods}

The smallness of the cross sections at stellar energies makes indirect methods quite useful in nuclear astrophysics. There are four often used indirect techniques: the asymptotic normalization coefficient (ANC) method [9], Coulomb breakup processes [7], the Trojan Horse [8] and Surrogate [23] methods.

In Sect. 3, we present an application of the ANC method, which is suitable for peripheral reactions, i.e. to capture reactions toward weakly bound states. Due to the low binding energy, this capture occurs well outside the nuclear range. In that case, the capture cross section $\sigma_{\gamma}$ can be written as

$$
\sigma_{\gamma}(E)=|C|^{2} f(E)
$$

where $C$ is the ANC of the bound state, and $f(E)$ a function which only depends on well known properties, such as the binding energy, masses and charges, angular momentum, etc. At very low energies, the knowledge of the cross section relies on the ANC only. A typical example is the ${ }^{7} \mathrm{Be}(\mathrm{p}, \gamma)^{8} \mathrm{~B}$ where the ground state is bound by $137 \mathrm{keV}$.

\section{Application to ${ }^{14} \mathbf{C}(\mathbf{n}, \gamma){ }^{15} \mathbf{C}$}

The main contribution to the ${ }^{14} \mathrm{C}(\mathrm{n}, \gamma){ }^{15} \mathrm{C}$ reaction rate comes from the direct $\mathrm{E} 1$ capture from the initial $p$-wave to the relatively weakly bound $\frac{1}{2}^{+}$ground state of ${ }^{15} \mathrm{C}$. This reaction is a good 
case for the ANC method. The neutron capture on a long-lived radioactive target ${ }^{14} \mathrm{C}$ provides one of the few possible test cases where a comparison between direct and indirect methods is possible. On the other hand, the ${ }^{14} \mathrm{C}(\mathrm{n}, \gamma){ }^{15} \mathrm{C}$ reaction is interesting on its own because of its important astrophysical applications.

Currently, a puzzling disagreement exists between the cross sections $\sigma_{n, \gamma}$ of ${ }^{14} \mathrm{C}(\mathrm{n}, \gamma){ }^{15} \mathrm{C}$ measured directly, determined indirectly and calculated theoretically. The first direct measurement in Ref. [24] provided $\sigma_{n, \gamma}=1.1 \pm 0.28 \mu \mathrm{b}$ which is about five times smaller than the theoretical value of $5.1 \mu \mathrm{b}$ predicted earlier in Ref. [25] within a potential model. The subsequent folding model calculations [26] and microscopic cluster model calculations [27] have confirmed the large value of Ref. [25]. Recently, the ${ }^{14} \mathrm{C}(\mathrm{n}, \gamma){ }^{15} \mathrm{C}$ cross sections have been determined indirectly in three dissociation experiments of ${ }^{15} \mathrm{C}[28,29,30]$.

In a recent work [10], we propose to use charge symmetry between ${ }^{15} \mathrm{C}\left(\frac{1}{2}^{+}\right)$and its isobar analog ${ }^{15} \mathrm{~F}\left(\frac{1}{2}^{+}\right)$as a strong and model-independent tool to discriminate between different determinations and predictions for the ${ }^{14} \mathrm{C}(\mathrm{n}, \gamma){ }^{15} \mathrm{C}$ cross sections. It has been shown recently that a simple relationship exists between the ANC of two analog levels [31]. This relationship has been extended to the case where one of the analog levels is unbound. In that case it is possible to link the ANC of the bound level with the width of the resonance in the mirror nucleus [32].The ratio

$$
\mathscr{R}_{\Gamma}=\Gamma_{p} / C_{n}^{2}
$$

where $\Gamma_{p}$ is the width of the proton unbound resonance, can be approximated by a model-independent analytical expression that essentially contains the neutron separation energy, and the energy $E_{R}$ of the proton resonance. This property is very helpful when one of the mirror nuclei is short-lived: a measurement of the ANC of the stable nucleus can indirectly provide the desired ANC. This simple relationship, suggested in [31] has been tested on different systems by a microscopic approach $[33,32]$ where both quantities are computed individually, and their ratio is compared with the simple analytical formula. The accuracy is in general better than $10 \%$ for narrow resonances.

This simple approach is used for the ${ }^{14} \mathrm{C}(\mathrm{n}, \gamma){ }^{15} \mathrm{C}$ reaction. The mirror system ${ }^{14} \mathrm{O}+\mathrm{p}$ is unbound, and has been recently investigated by elastic scattering with a radioactive ${ }^{14} \mathrm{O}$ beam [34]. A microscopic cluster model is able to reproduce fairly well the data [35]. As the ${ }^{15} \mathrm{~F}$ ground state is fairly broad, we have used the same idea, but with a more precise calculation. The ratio (3.1) has been determined within the microscopic approach [35]. Under different conditions of calculation, we have checked that this ratio remains nearly constant. In Fig.1, we present $\sigma_{n, \gamma} E^{-1 / 2}$ derived from mirror symmetry with the resonance properties derived in Ref. [35] by the dark area. The light area corresponds to calculations with other ${ }^{15} \mathrm{~F}$ properties. Our predictions agree with indirect determinations from Refs. [29, 30] but they do not leave any room for small $\sigma_{n, \gamma}$. The charge symmetry of the ${ }^{15} \mathrm{C} \rightarrow{ }^{14} \mathrm{C}+\mathrm{n}$ and ${ }^{15} \mathrm{~F} \rightarrow{ }^{14} \mathrm{O}+\mathrm{p}$ decays significantly reduces the uncertainty in the current knowledge of the ${ }^{14} \mathrm{C}(\mathrm{n}, \gamma){ }^{15} \mathrm{C}$ cross sections and favors the earlier theoretical predictions for this reaction from Ref. [25]. It also shows that directly and some indirectly measured cross sections in $[24,28,36]$ contradict charge symmetry in the ${ }^{15} \mathrm{C}-{ }^{15} \mathrm{~F}$ mirror pair ${ }^{1}$. This contradiction deserves thorough attention because it brings into question the determination of the astrophysically important $(\mathrm{n}, \gamma)$ cross sections for short-lived radioactive targets.

\footnotetext{
${ }^{1}$ The data of Ref. [36] are however under reanalysis and are probably underestimated [37].
} 


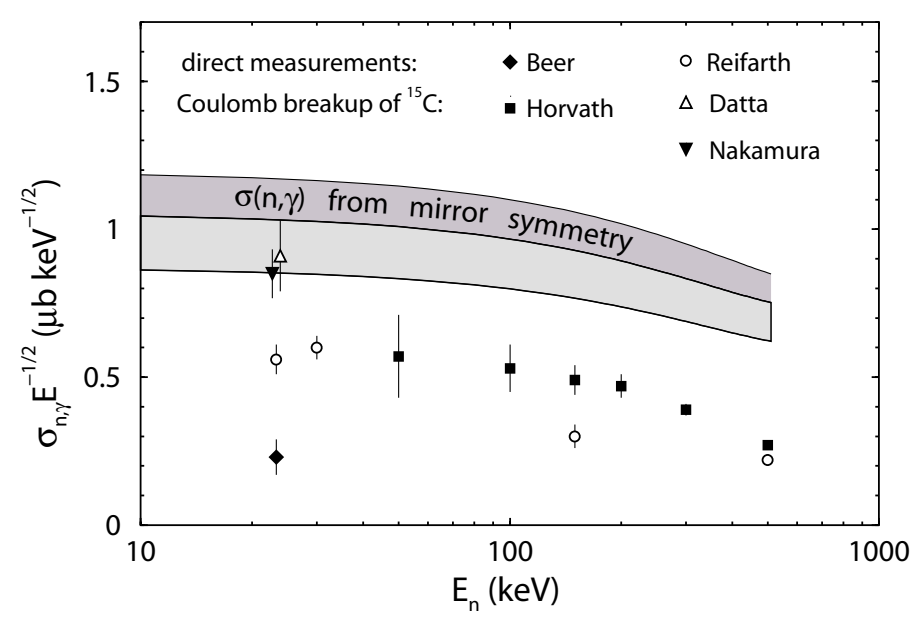

Figure 1: Experimental (data points) and theoretical values of $\sigma_{n, \gamma} E^{-1 / 2}$. The dark area corresponds to $\sigma_{n, \gamma}$ derived from mirror symmetry assuming $E_{R}$ and $\Gamma_{p}$ from Ref. [35] while the light one corresponds to all other $E_{R}$ and $\Gamma_{p}$.

\section{A microscopic approach to the ${ }^{18} \mathbf{F}(\mathbf{p}, \alpha){ }^{15} \mathbf{O}$ reaction}

\subsection{Introduction}

The ${ }^{18} \mathrm{~F}(\mathrm{p}, \alpha){ }^{15} \mathrm{O}$ reaction plays an important role in novae nucleosynthesis [11]. The typical temperature range in novae is of the order of $1-4 \times 10^{8} \mathrm{~K}$. Consequently, ${ }^{19} \mathrm{Ne}$ resonances of importance in the burning process are located in an interval of about $1 \mathrm{MeV}$ above the ${ }^{18} \mathrm{~F}+\mathrm{p}$ threshold $(Q=6.41 \mathrm{MeV})$. Many uncertainties remain on the determination of the ${ }^{18} \mathrm{~F}(\mathrm{p}, \alpha){ }^{15} \mathrm{O}$ reaction rate at these energies. Until now, the astrophysical $S$-factor has been calculated as a sum of contributions of individual states. Among those resonances, only two of them seem to be reasonably well understood. A $3 / 2^{+}$state at $E_{x}=7.07 \mathrm{MeV}\left(E_{c m}=0.66 \mathrm{MeV}\right)$ is an $s$ wave and is known to be a single particle state, with a fairly large spectroscopic factor [38]. It has been widely investigated in the literature, through transfer (see Ref. [39] and references therein) or elastic [40, 41] reactions, or with indirect methods (see Refs. [42, 43] and references therein). On the other hand, the existence of a $3 / 2^{-}$state at $E_{c m}=0.33 \mathrm{MeV}$ [44] seems now well established, and should play a role for temperatures $T_{9} \approx 0.3-0.4$. Here we briefly present a recent work on a microscopic study of the ${ }^{18} \mathrm{~F}(\mathrm{p}, \alpha){ }^{15} \mathrm{O}$ reaction at stellar energies [45].

\subsection{Brief description of the model}

The wave functions are defined in the Generator Coordinate Method (GCM) which assumes a cluster structure for the $A$-nucleon system [15]. In a given partial wave $J M \pi$, the wave function of the system is given by a superposition of ${ }^{18} \mathrm{~F}+\mathrm{p},{ }^{15} \mathrm{O}+\alpha$ and ${ }^{18} \mathrm{Ne}+\mathrm{n}$ cluster wave functions, each channel involving various excited states (see Ref. [45] for details). More precisely the wave function is given by

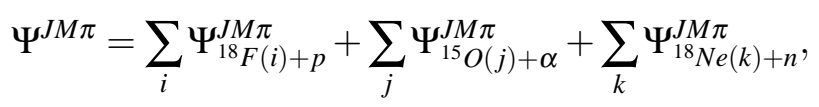


where the sums run over all internal states. We use a recently developed nucleon-nucleon interaction [45] which allows to constrain a threshold, and to keep one parameter to reproduce some important energy of the system, such a the energy of a resonance, for example. The interaction reproduces the energy of the $3 / 2^{+}$resonance in ${ }^{19} \mathrm{Ne}\left(E_{x}=7.07 \mathrm{MeV}, E_{c m}=0.66 \mathrm{MeV}\right)$ which is well known to be a single-particle state [38], accurately described by a ${ }^{18} \mathrm{~F}+\mathrm{p}$ model. It also reproduces the $Q$ value.

\subsection{Spectroscopic properties of ${ }^{19} \mathrm{~F}$ and ${ }^{19} \mathrm{Ne}$}

Our goal is essentially to provide a general discussion of the ${ }^{18} \mathrm{~F}(\mathrm{p}, \alpha){ }^{15} \mathrm{O}$ cross section, in the framework of a microscopic approach. Such a model is of course not expected to provide an accurate description of all resonances. Consequently, we focus on two specific partial waves, $J=1 / 2^{+}$and $J=3 / 2^{+}$, which correspond to $s$ waves, expected to dominate the cross section at low energies. As an example the level schemes for $J=1 / 2^{+}$is given in Fig. 2. Energies are given from the ${ }^{18} \mathrm{~F}+\mathrm{n}$ and ${ }^{18} \mathrm{~F}+\mathrm{p}$ threshold for ${ }^{19} \mathrm{~F}$ and ${ }^{19} \mathrm{Ne}$, respectively.

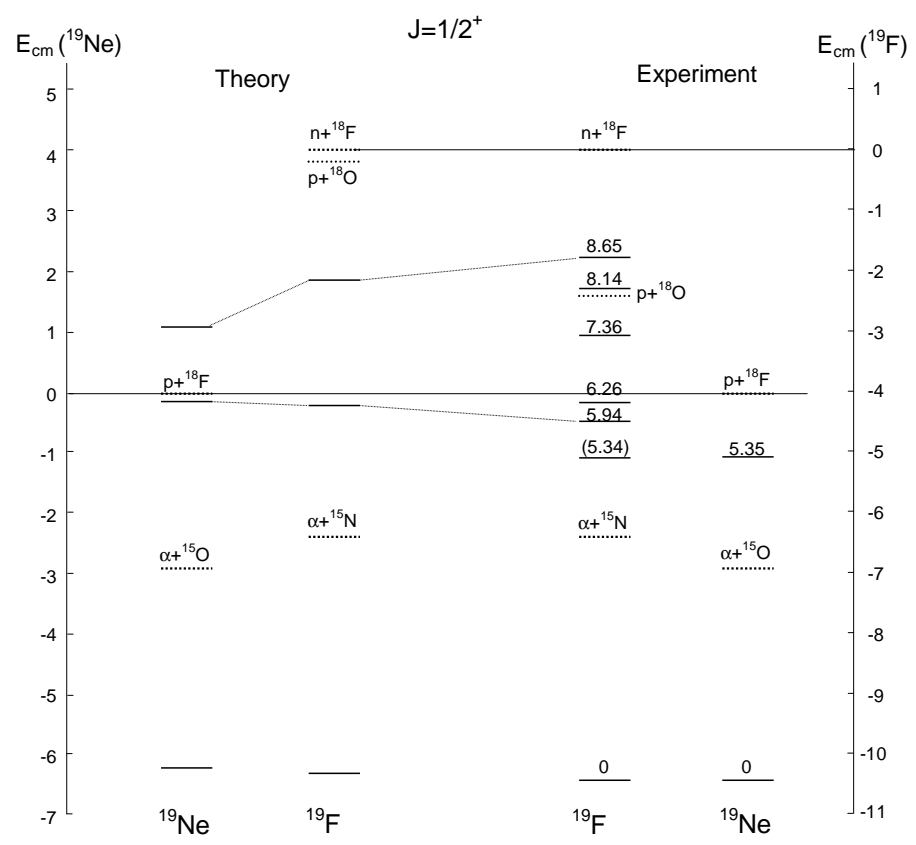

Figure 2: ${ }^{19} \mathrm{~F}$ and ${ }^{19} \mathrm{Ne}$ spectra for $J=1 / 2^{+}$. Energies are in $\mathrm{MeV}$. The thresholds are displayed as dotted lines.

The GCM energies of the ground states are in nice agreement with experiment (within 0.2 $\mathrm{MeV}$ ). The second GCM $1 / 2^{+}$state presents a dominant component in the $\alpha+{ }^{15} \mathrm{~N} / \alpha+{ }^{15} \mathrm{O}$ channel. Experimentally, the $5.94 \mathrm{MeV}$ state in ${ }^{19} \mathrm{~F}$ is known to be an $\alpha+{ }^{15} \mathrm{~N}$ state, and has been described by previous single-channel $\alpha+{ }^{15} \mathrm{~N}$ cluster models [46]. The GCM gives rise to a third $1 / 2^{+}$resonance, at $E_{x}=8.3 \mathrm{MeV}$. Its width $\left(\Gamma_{G C M}=180 \mathrm{keV}\right)$ suggests that the experimental counterpart is the $8.65 \mathrm{MeV}$ state $\left(\Gamma_{\exp } \approx 300 \mathrm{keV}\right)$. The predicted energy is quite close to the experimental value.

The study of $1 / 2^{+}$levels provides two conclusions:

(i) In ${ }^{19} \mathrm{Ne}$, the third GCM level, analog of the $8.65 \mathrm{MeV}$ state in ${ }^{19} \mathrm{~F}$, is located at lower energy, 
near $1 \mathrm{MeV}$ above the ${ }^{18} \mathrm{~F}+\mathrm{p}$ threshold. Therefore it might play a role in the ${ }^{18} \mathrm{~F}(\mathrm{p}, \alpha){ }^{15} \mathrm{O}$ reaction. The large Coulomb shift supports an important spectroscopic factor. From theoretical arguments, this state can be considered as a single-particle state, similar to the $3 / 2^{+}(7.07 \mathrm{MeV})$ level in ${ }^{19} \mathrm{Ne}$. Although it should play a role in the cross section, as $s$-wave resonance, it has not been clearly observed in ${ }^{19} \mathrm{Ne}$.

(ii) Comparing the theoretical and experimental ${ }^{19} \mathrm{~F}$ spectra indicates that some levels are missing in the GCM. Although they are not observed in ${ }^{19} \mathrm{Ne}$ they might also contribute to the ${ }^{18} \mathrm{~F}(\mathrm{p}, \alpha){ }^{15} \mathrm{O}$ $S$-factor. As they are not described by the present two-cluster model, which includes many configurations, it is likely that these states should have a more complicated structure, such as a three-body structure. It is consequently reasonable to assume that their spectroscopic factors in $p$ and $\alpha$ channels should be small, and that their role in the cross section should be of minor importance.

From an experimental point of view, many $3 / 2^{+}$states are known in ${ }^{19} \mathrm{~F}$ up to $8 \mathrm{MeV}$. Although the cluster theory does reproduce three of them (the ground state, the $\alpha$-cluster molecular resonance, and the single-particle state at $7.07 \mathrm{MeV}$ ), some other states are missing. An obvious drawback of the model is that the $3 / 2^{+}$doublets near $6.5 \mathrm{MeV}$ cannot be described, although their states are very close to the ${ }^{18} \mathrm{~F}+\mathrm{p}$ threshold in ${ }^{19} \mathrm{Ne}$. This problem is well known in cluster models: it arises from the limited number of configurations. When a resonance is missing in the GCM, it should correspond to other cluster configurations, such as three-body structures or other two-body arrangements. This means that the ${ }^{18} \mathrm{~F}+\mathrm{p}$ and $\alpha+{ }^{15} \mathrm{O}$ configurations, included in the model, should play a less important role, and that the spectroscopic factors in these channels should be quite small.

\subsection{The ${ }^{18} \mathbf{F}(\mathbf{p}, \alpha){ }^{15} \mathrm{O}$ reaction}

The GCM $S$-factor is shown in Fig. 3. Near the $3 / 2^{+}$resonance, the agreement with the data is excellent, as only the energy has been fitted by the $N N$ interaction. Below $0.3 \mathrm{MeV}$, the $3 / 2^{+}$ contribution presents an interference pattern, due to other $3 / 2^{+}$resonances. The $1 / 2^{+}$contribution is essentially determined by the properties of a broad resonance near $1 \mathrm{MeV}$, and of a subthreshold state (see Fig. 2).

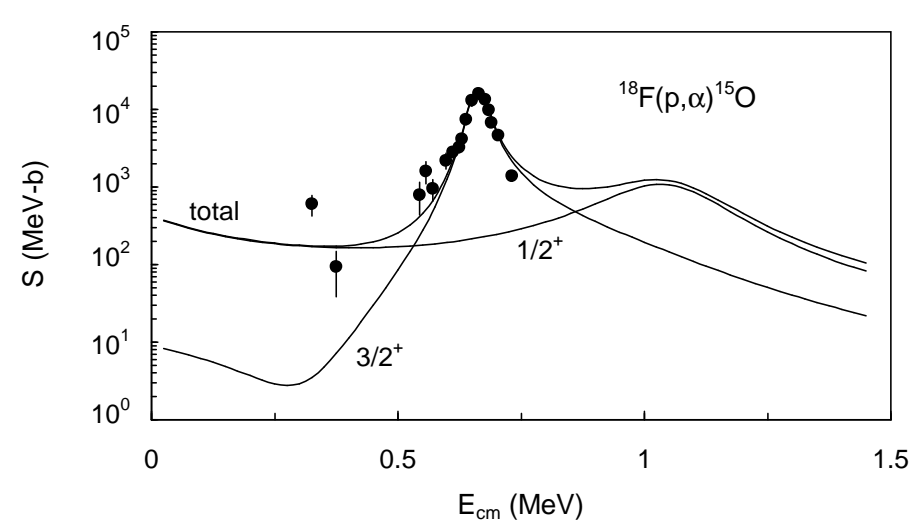

Figure 3: ${ }^{18} \mathrm{~F}(\mathrm{p}, \alpha){ }^{15} \mathrm{O} S$-factor for $J=1 / 2^{+}$and $J=3 / 2^{+}$. The experimental data are taken from Ref. [39, 44].

The GCM $S$-factor shown in Fig. 3 is of course a good estimate of the data, but some resonances are missing, and others are not located at the experimental energy. To improve the accuracy 
on the theoretical $S$-factor, we have combined GCM information with experimental data. This procedure has been successfully used in other reactions, such as ${ }^{12} \mathrm{C}(\alpha, \gamma){ }^{16} \mathrm{O}$ for example [47]. Let us start with the $1 / 2^{+}$contribution. As shown in Fig. 3, two states determine the $S$-factor. These states are known experimentally in ${ }^{19} \mathrm{~F}$, but are not observed in ${ }^{19} \mathrm{Ne}$. It is reasonable to assume that the difference between the GCM and the data is identical for the mirror nuclei. The ${ }^{19} \mathrm{Ne}$ energies have been therefore corrected by this difference (providing $-0.4 \mathrm{MeV}$ and $1.5 \mathrm{MeV}$ ), and the partial widths unknown in ${ }^{19} \mathrm{~F}$ are modified accordingly.

For the $3 / 2^{+}$partial wave, a similar procedure has been used. We have complemented the microscopic $R$-matrix calculation with both states of the doublet $\left(E_{c m}=8 \mathrm{keV}\right.$ and $\left.38 \mathrm{keV}\right)$. Partial widths have been taken from the literature. As the interference signs are not known, we have considered the four possibilities. This procedure provides the different curves in Fig. 4. Within the resonance, the theory slightly underestimates the data. This could be compensated by a slight readjustment of the partial widths, but our goal here is a qualitative discussion, rather than an accurate fit of the data. The main conclusion from Fig. 4 is that, in the temperature range typical of novae (corresponding to $E_{c m} \approx 0.2-0.3 \mathrm{MeV}$ ) the uncertainties of the $S$-factor due to the interference patterns is much smaller than expected [48]. The reason is that the main contribution at nova energies comes from the $1 / 2^{+}$partial wave.

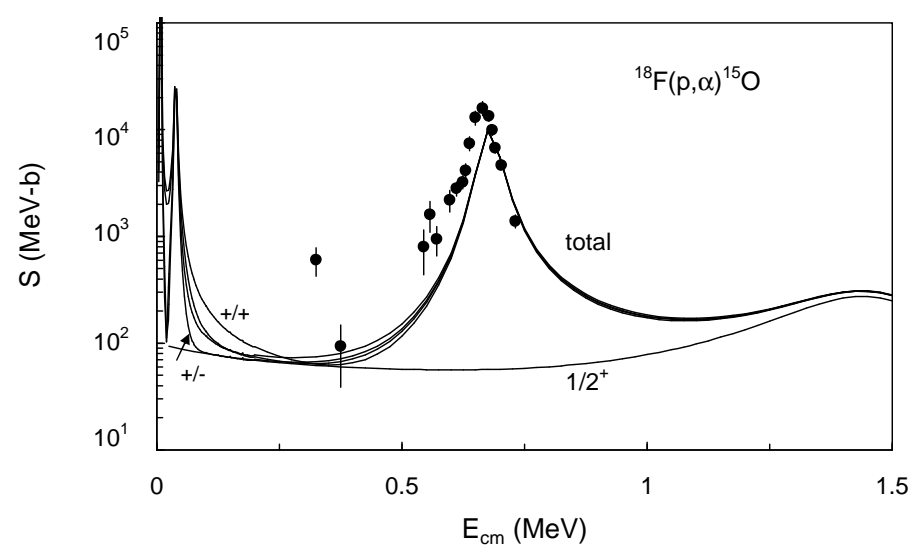

Figure 4: Corrected ${ }^{18} \mathrm{~F}(\mathrm{p}, \alpha){ }^{15} \mathrm{O} S$-factor for $J=1 / 2^{+}$and $J=3 / 2^{+}$(see text). The signs correspond to different interference signs in the $3 / 2^{+}$contribution.

The main novelty of this work deals with new $1 / 2^{+}$states near the proton threshold in ${ }^{19} \mathrm{Ne}$. Experimentally, this state could be observed by elastic scattering. It is interesting to point out that, in a ${ }^{18} \mathrm{~F}+\mathrm{p}$ elastic-scattering experiment, Bardayan et al. [41] mention the need for a broad $s$ state to fit their cross section. The experiment was however limited to energies $E_{c m}<1.01 \mathrm{MeV}$, and a clear identification could not be performed. We present in Fig. 5 the theoretical scattering cross section at $\theta_{c m}=180^{\circ}$ (corresponding to $\theta_{l a b}=0^{\circ}$ in reverse kinematics). This cross section should be considered as qualitative only, as only the $1 / 2^{+}$and $3 / 2^{+}$partial waves have been included (for other partial waves, up to $\ell=2$ we take the hard-sphere approximation of the phase shift). The main emphasis should be put on the difference between the cross sections with and without the broad $1 / 2^{+}$state. It presence provides a clear signature in the cross section, and should be observable in current experiments. 


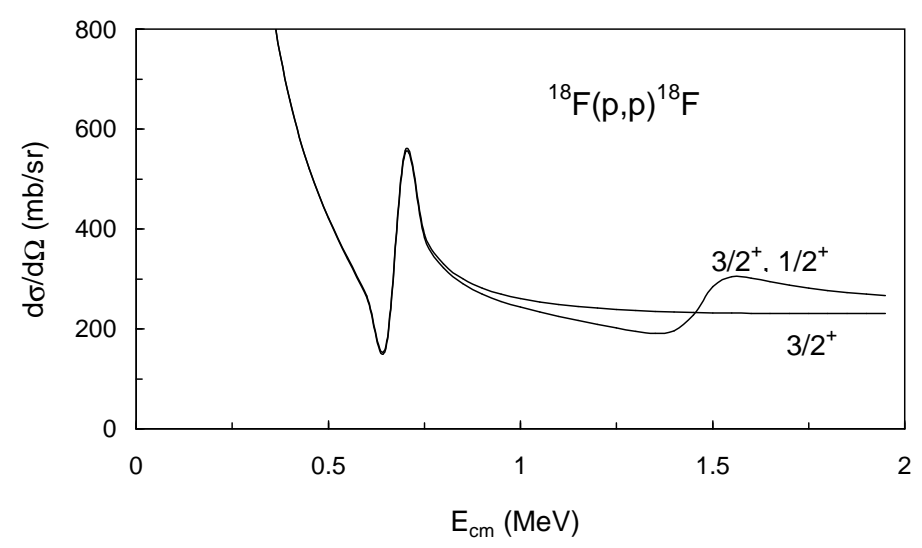

Figure 5: ${ }^{18} \mathrm{~F}+\mathrm{p}$ elastic cross section at $\theta_{c m}=180^{\circ}$, with the $3 / 2^{+}$partial wave only, and with the addition of $J=1 / 2^{+}$.

\section{Conclusion}

In this work, we have given an short overview of some models relevant for in nuclear astrophysics. We were only concerned with reactions, without discussing other aspects, such as masses, beta decays, etc. In general, charged-particle induced reactions occur at energies much lower than the Coulomb barrier, and the corresponding cross sections are therefore extremely small. Another characteristic is that there is almost no systematics. In the low-mass region, each reaction presents its own peculiarities and difficulties, in the theoretical as well as in the experimental viewpoints.

A very impressive amount of work has been devoted to nuclear astrophysics in the last decades. Although most reactions involving light nuclei are sufficiently known, some reactions, such as ${ }^{18} \mathrm{~F}(\mathrm{p}, \alpha){ }^{15} \mathrm{O}$ still require much effort to reach the accuracy needed for stellar models.

\section{References}

[1] C. Rolfs and W.S. Rodney, Cauldrons in the Cosmos, University of Chicago Press, 1988.

[2] M. Junker et al., Phys. Rev. C57 (1998) 2700.

[3] M.S. Smith and K.E. Rehm, Annu. Rev. Nucl. Part. Sci. 51 (2001) 91.

[4] P. Descouvemont, Theoretical models for nuclear astrophysics, Nova Science, 2003.

[5] P. Descouvemont and T. Rauscher, Nucl. Phys. A777 (2006) 137.

[6] A.M. Mukhamedzhanov et al., Eur. Phys. J. A27 (Suppl. 1) (2006) 205.

[7] G. Baur et al., Nucl. Phys. A458 (1986) 188.

[8] C. Spitaleri et al., Phys. Rev. C9 (2004) 055806.

[9] A.M. Mukhamedzhanov et al., Phys. Rev. C67 (2003) 065804.

[10] N.K. Timofeyuk et al., Phys. Rev. Lett. 96 (2006) 162501.

[11] A. Coc, M. Hernanz, J. José, and J.-P. Thibaud, Astron. Astrophys. 357 (2000) 561.

[12] A.M. Lane and R.G. Thomas, Rev. Mod. Phys. 30 (1958) 257. 
[13] B. Buck, H. Friedrich, and C. Wheatley, Nucl. Phys. 275 (1977) 246.

[14] G.R. Satchler, Direct Nuclear Reactions, Oxford, 1983.

[15] K. Wildermuth and Y.C. Tang, A Unified Theory of the Nucleus, ed. by K. Wildermuth and P. Kramer, Vieweg, Braunschweig (1977).

[16] P. Navratil, C.A. Bertulani, and E. Caurier, Phys. Lett. B634 (2006) 191.

[17] T. Rauscher, F.-K. Thielemann, and K.-L. Kratz, Phys. Rev. C56 (1997) 1613.

[18] L. Buchmann et al., Phys. Rev. C54 (1996) 393.

[19] M.J. Jamieson, Comp. Phys. Comm. 125 (2000) 193.

[20] M. Dufour and P. Descouvemont, Nucl. Phys. A605 (1996) 160.

[21] D. Baye and M. Kruglanski, Phys. Rev. C45 (1992) 1321.

[22] P. Descouvemont, Nucl. Phys. A596 (1996) 285.

[23] W. Younes and H.C. Britt, Phys. Rev. C67 (2003) 024610.

[24] H. Beer et al., Astrophys. J. 387 (1992) 258.

[25] M. Wiescher, J. Görres and F.K. Thielemann, Astrophys. J. 363 (1990) 340.

[26] H. Herndl et al., Phys. Rev. C60 (1999) 064614.

[27] P. Descouvemont, Nucl. Phys. A675 (2000) 559.

[28] Á. Horváth et al., Astrophys. J. 570 (2002) 926.

[29] U. Datta Pramanik and LAND-CB-FRS-Collaboration, Prog. Theor. Phys. Suppl. 146 (2002) 427.

[30] T. Nakamura et al., Nucl.Phys. $\mathbf{A 7 2 2}$ (2003) 301c, and T. Nakamura, private communication.

[31] N.K. Timofeyuk, R.C. Johnson and A.M. Mukhamedzhanov, Phys. Rev. Lett. 91 (2003) 232501.

[32] N.K. Timofeyuk and P. Descouvemont, Phys. Rev. C72 (2005) 064324.

[33] N.K. Timofeyuk and P. Descouvemont, Phys. Rev. C71 (2005) 064305.

[34] V.Z. Goldberg et al., Phys. Rev. C69 (2004) 031302(R).

[35] D. Baye, P. Descouvemont and F. Leo, Phys. Rev. C72 (2005) 024309.

[36] R. Reifarth et al., Nucl. Phys. A758 (2005) 787c.

[37] K. Sümmerer, private communication.

[38] H.T. Fortune and R. Sherr, Phys. Rev. C61 (2000) 024313.

[39] D.W. Bardayan et al., Phys. Rev. C63 (2001) 065802.

[40] J.-S. Graulich et al., Phys. Rev. C63 (2001) 011302.

[41] D.W. Bardayan et al., Phys. Rev. C70 (2004) 015804.

[42] N. de Séréville et al., Phys. Rev. C67 (2003) 052801.

[43] R.L. Kozub et al., Phys. Rev. C73 (2006) 044307.

[44] D.W. Bardayan et al., Phys. Rev. Lett. 89 (2002) 262501.

[45] M. Dufour and P. Descouvemont, to be published .

[46] T. Sakuda and F. Nemoto, Prog. Theor. Phys. 62 (1979) 1274; Prog. Theor. Phys. 62 (1979) 1606.

[47] P. Descouvemont and D. Baye, Phys. Rev. C36 (1987) 1249.

[48] N. de Séréville, E. Berthoumieux, and A. Coc, Nucl. Phys. A758 (2005) 745c. 